From the authors:

We welcome the correspondence submitted by P.H. Quanjer and colleagues addressing the recently published American Thoracic Society (ATS)/European Respiratory Society (ERS) statement on research questions in chronic obstructive pulmonary disease (COPD). The issue they raise has been a matter of continuous debate in the literature, symposia, and national and international meetings. It centres on whether to use the fixed ratio of forced expiratory volume in $1 \mathrm{~s}$ (FEV1)/forced vital capacity (FVC) of $<0.7$ or the lower limit of normal (LLN) for the diagnosis of COPD.

In this regard, the ATS/ERS document is very clear $[1,2]$. It begins this section on diagnosis by stating that the diagnosis is confirmed by the presence of airflow limitation that is not fully reversible and is defined by a low post-bronchodilator FEV1/FVC ratio. This is not controversial or equivocal and this is a statement that we can all agree upon. Subsequently, it presents a summary of the arguments used in the controversy that takes no side. "The threshold FEV1/FVC ratio that should be used to confirm an airflow limitation is uncertain. A post-bronchodilator FEV1/FVC ratio of less than 0.7 has traditionally been the criterion for airflow limitation. This threshold may result in more frequent identification of airflow limitation and, hence, diagnosis of COPD among the elderly and less frequent diagnosis among young adults less than 45 years of age compared with a threshold based on the [LLN] of FEV1/FVC. Although these observations are particularly relevant for epidemiological studies, advocates for the fixed ratio argue that it identifies a number of patients with significant pulmonary pathology and respiratory morbidity not detected by the LLN and advocates for the LLN argue that the fixed ratio is more likely to yield false-positive results". It then presents research questions aimed at helping provide guidance for research in this area. Is this not what P.H. Quanjer and colleagues would like to see?

However, I would like to remind P.H. Quanjer and colleagues that there should be no confusion between the definition of airflow limitation and that of COPD. Airflow limitation represents a physiological abnormality, with an arbitrary threshold placed at an operational level. COPD is a disease, which encompasses a much wider concept. For the record, I would like to refer P.H. Quanjer and colleagues to the excellent article written by a pulmonologist, J.G. Scadding, entitled: "Health and disease: what can medicine do for philosophy?" [3]. Disease is defined as a "sum of abnormal phenomena displayed by a group of organisms in association with a specified common characteristic or several characteristics by which they differ from the norm of their species in such a way to place them at a biological disadvantage". The defining characteristics of a disease are four. 1) Clinical description or syndrome: the oldest of all are the symptoms and signs of the disease in question. 2) Structural abnormalities: in the case of COPD, airway remodelling and emphysema. 3) Disorders of function: where an abnormal spirometric finding would be included. New biological and imaging information is offering novel insights that can help modify definitions. 4) Causation or aetiology: in the specific case of COPD, cigarette smoking, biomass exposure and $\alpha_{1}$-antitrypsin. In this context, abnormal spirometry does not establish the presence of the disease, as categorically stated in the opening sentence of the second paragraph of P.H. Quanjer and colleagues' correspondence. Abnormal spirometry (with an operational definition yet to be fully agreed upon) only confirms the presence of airflow limitation and fulfils the second (but not the only or most important) characteristic of a disease.

Finally, we would like to remind P.H. Quanjer and colleagues that the published statement is not a guideline and was developed to help guide the research endeavour that the whole community interested in COPD will conduct over the ensuing years.

@ERSpublications

Abnormal spirometry confirms airflow limitation but is not the only or most important characteristic of COPD http://ow.ly/RVXxW

Bartolome R. Celli for the authors of the ATS/ERS statement on research questions in COPD Harvard Medical School, Brigham and Women's Hospital, Boston, MA, USA.

Correspondence: Bartolome R. Celli, Pulmonary and Critical Care Division, Brigham and Women's Hospital, 75 Francis Street, Boston, MA 02115, USA. E-mail: bcelli@partners.org

Received: Aug 172015 | Accepted after revision: Sept 012015

Conflict of interest: Disclosures can be found alongside the online version of this article at erj.ersjournals.com

\title{
References
}

1 Celli BR, Decramer M, Wedzicha JA, et al. An Official American Thoracic Society/European Respiratory Society statement: Research questions in chronic obstructive pulmonary disease. Am J Respir Crit Care Med 2015; 191: e4-e27. 


\section{We must join forces in the battle against COPD}

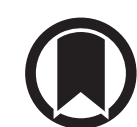

CrossMark

\section{To the Editor:}

The recently published American Thoracic Society (ATS)/European Respiratory Society (ERS) statement on research questions in chronic obstructive pulmonary disease (COPD) [1] is an excellent and extensive document. It will, without doubt, contribute to better understanding of COPD and hopefully direct research to important unmet needs. Our only disappointment is that the authors did not include significant representation from primary care or public health: disappointing because globally most people with COPD are diagnosed and managed in the community by primary care clinicians, and many of the unmet individual and population research needs can only be defined and answered by professionals working in these settings.

5 years ago, the International Primary Care Respiratory Group (IPCRG) published a research needs statement written by practising primary care clinicians from 22 countries with a range of socioeconomic backgrounds [2]. We identified 145 research questions to set the agenda for primary care research in the context of diagnosis and management of asthma, allergic rhinitis, COPD, tobacco dependence and respiratory infections. This was followed in 2012 by a Delphi exercise in which 22 global participants prioritised the questions based on their clinical importance, feasibility and international relevance. This generated a list of seven research questions that achieved total agreement and 24 that reached the consensus threshold of $90 \%$ [3]. The themes we identified 3 years ago remain relevant today. Many are echoed in the ATS/ERS statement: how a prompt diagnosis of a respiratory disease can be suspected in a consultation and the condition assessed; and effective approaches to implementing self-management, adjusting treatment regimens and encouraging treatment adherence. We too highlighted that multimorbidity is the norm in people with COPD [4], and called for research to define the approach to care for a multimorbid patient who may have a range of physical and mental health problems (specifically including tobacco dependency), and who is at risk from polypharmacy [5].

However, the IPCRG Delphi process also prioritised other, very basic questions: what are the "simple" tools and pragmatic approaches for diagnosing and assessing patients in the low-tech community environment; how can guidelines be implemented; and how do we educate and support clinicians to tackle chronic respiratory diseases? These questions are absent from this ERS/ATS statement and yet without addressing these implementation science questions, there will be no significant improvement in COPD diagnosis and care.

In addition, any statement of research needs for COPD needs to be set in the context of the noncommunicable disease epidemic, and reflect that most new cases will be in low- and middle-income countries. The World Health Organization estimates that 2.4 billion people, generally among the world's poorest, rely on biomass fuels for their heating and cooking [6]. The IPCRG has highlighted in its FRESH AIR work the significance of biomass fuels as a cause of COPD, often in relatively young populations: $40 \%$ of the people with COPD in our Ugandan project were $<40$ years of age [7]. This is a priority for our international group with a reach of 125000 primary care clinicians worldwide. The only way to address such challenges is a population health approach to reduce smoking and exposure to biomass smoke. Furthermore, the global economic cost of COPD is US\$2.1 trillion [8]. Governments and their healthcare systems need research to enable them to understand the most cost-effective interventions for populations, and how to achieve the greatest value from drug and nondrug healthcare interventions.

The IPCRG called for research "undertaken within primary care, recruiting participants representative of primary care populations, evaluating interventions realistically delivered over appropriate timescales within primary care, and drawing conclusions that will be meaningful to professionals working within primary care" [3]. Bridging the second translational gap, such research will generate evidence relevant to the care of people with COPD in their communities. 